\title{
Emodin sensitizes human pancreatic cancer cells to EGFR inhibitor through suppressing Stat3 signaling pathway
}

This article was published in the following Dove Press journal: Cancer Management and Research

\author{
Zhaohong Wang $\mathbb{B}^{1,2, *}$ \\ Hui Chen ${ }^{1}$ * \\ Jingjing Chen ${ }^{1} *$ \\ Zhong Hong $\mathbb{D}^{\prime}$ \\ Yi Liao (1) \\ Qiyu Zhang ${ }^{1,2}$ \\ Hongfei Tong'
}

'Department of Hepatobiliary Surgery, The Second Affiliated Hospital and Yuying Children's Hospital of Wenzhou Medical University, Wenzhou 325000, People's Republic of China; ${ }^{2}$ Department of Hepatobiliary Surgery, The First Affiliated Hospital of Wenzhou Medical University, Wenzhou 325000, People's Republic of China

*These authors contributed equally to this work
Background: Excessive expression of EGFR is closely related to tumor formation, transfer and deterioration, which has attracted much attention. EGFR overexpression may be detected in up to $90 \%$ of pancreatic tumors. However, drug resistance of EGFR inhibitors targeting treatment severely limits its clinical application.

Methods: In this study, Western blotting was used to detect the expression of p-Stat3, EGFR, Bcl-2, cleaved-caspase3 and Bax. Cell apoptosis was evaluated via flow cytometry. The colon assay and MTT assay were applied for detecting the cell proliferation in vitro. The xenograft mouse model was used to examine the cell proliferation in vivo.

Results: Emodin remarkably enhanced the anti-cancer effect of EGFR inhibitor on pancreatic cancer cells. In addition, emodin promoted afatinib-induced apoptosis by inhibiting the Stat3 signaling pathway. Meanwhile, siRNAs against Stat3 significantly increased the apoptosis of pancreatic cancer cells. EGFR inhibitor promoted phosphorylation of Stat3 in pancreatic cancer cells. Interestingly, emodin combined with EGFR inhibitor inhibited the proliferation of pancreatic cancer cells in vitro. The tumor xenograft mice model was further confirmed that emodin possessed a synergy anticancer effect with afatinib on pancreatic cancer cells by regulating the Stat3 expression.

Conclusion: These results indicate that the combination of emodin with EGFR inhibitor is an effective therapeutic strategy to sensitize human pancreatic cancer.

Keywords: pancreatic cancer, EGFR inhibitors, afatinib, Stat3

\section{Introduction}

Pancreatic cancer is one of the most aggressive human malignancies with a high mortality rate. Surgical resection is the preferred treatment for pancreatic cancer, but less than $20 \%$ of patients are eligible for the operation with poor prognosis. ${ }^{1-3}$ Chemotherapy is still essential in the treatment of advanced pancreatic cancer, preventing postoperative recurrence and prolonging the survival of patients. ${ }^{4}$ The EGFR signaling pathway has been reported to play an important role in the proliferation, apoptosis, differentiation, migration and angiogenesis of pancreatic cancer cells. ${ }^{5}$ Gemcitabine-based chemotherapy combinations are the cornerstones of pancreatic cancer treatment, but the efficacy is not optimistic. ${ }^{6}$ The EGFR overexpression is closely associated with the formation, metastasis and deterioration of tumors, which has become a hot target in recent years. ${ }^{7}$ Based on their target, targeted therapeutic drugs against EGFR can be divided into two categories: one is small molecule EGFR-tyrosine kinase inhibitors (EGFR-TKIs) such as
Correspondence: Qiyu Zhang;

Hongfei Tong

Department of Hepatobiliary Surgery,

The Second Affiliated Hospital and Yuying

Children's Hospital of Wenzhou Medical

University, 109 Xueyuan W Road,

Lucheng Qu, Wenzhou Shi, Zhejiang

Sheng 32500, People's Republic of China

Tel +86 5778800 27I0;

+8657788002710

Email qiyuz@I26.com;

tonghongfei@I63.com 
erlotinib, gefitinib and afatinib, etc. ${ }^{8,9}$ the other is a EGFR-monoclonal antibody (EGFR-mAb) that specifically binds to the extracellular EGFR cells including cetuximab. ${ }^{10}$ However, the emergence of drug resistance in EGFR inhibitor targeted therapy has severely limited its clinical application. Further research on exploring the drug resistance mechanism of EGFR inhibitors and finding ways to overcome or reverse drug resistance is an urgent problem to be solved in the field of pancreatic cancer treatment, which is also the main purpose of this project.

Natural products have been found to play an important role in various diseases in recent years. And more reports pointed out that natural products suppress the cancer proliferation via different mechanisms. Bitter melon extract inhibits breast cancer growth in the preclinical model by induction of autophagy and modulation of the AMPK/mTOR pathway. ${ }^{11}$ And bitter melon extract inhibits head and neck squamous cell carcinoma growth by inhibiting FoxP3+ populations in the tumors and in spleens. $^{12}$

Emodin (1,3,8-trihydroxy-6-methyl-anthraquinone) as a natural product, an effective monomer isolated from the genus Rhubarb, Amaranthus and Senna, ${ }^{13}$ exerts extensive pharmacological effects, such as immune regulation, ${ }^{14}$ anti-inflammatory $^{15}$ and antitumor. ${ }^{16}$ Besides, emodin represents a crucial role in resisting various tumor cells by inhibiting tumor cell proliferation, invasion, metastasis, angiogenesis and promoting apoptosis. ${ }^{17,18}$ It has been determined that emodin inhibited the proliferation and promoted apoptosis of breast cancer cells by inhibiting the proto-oncogene human epidermal growth factor receptor-2 (HER-2) signaling pathway and endoplasmic reticulum stress and through $\mathrm{Ca} 2+$-dependent mitochondria. ${ }^{19}$ Two 2D quantitative structure-activity relationship (QSAR) models and molecular docking simulation indicated the possible binding site between emodin analogs and EFGR proteins. And corresponding results demonstrated the benefits of further investigations on the development of emodin analogs with improved anticancer bioactivity. However, the specific association between them is still worthy of further study. ${ }^{20}$ Moreover, emodin could induce apoptosis of colon cancer cells and exhibit potent anticancer activity mainly through its endogenous apoptotic pathway. ${ }^{21}$ It has also been proved that emodin directly inhibited the TGF protein expression in pancreatic cancer cells, leading to a decrease in the mRNA level of angiogenic factor-related genes, thereby inhibiting the occurrence and metastasis of tumors. ${ }^{22}$ In addition, emodin can synergize with a variety of antitumor drugs, enhance the sensitivity of tumor cells to radiotherapy and reduce its side effects, thus having a potential clinical application. ${ }^{23}$

In this study, we found that emodin combined with EGFR inhibitor afatinib in pancreatic cancer was superior to emodin or EGFR inhibitor alone in inhibiting cell proliferation, yet the specific mechanism of action between them requires further study. Recent studies have reported that Stat3 were important downstream regulators of the EGFR signaling pathway, which were involved in EGFR-mediated proliferation and differentiation of cancer cells and participated in regulating diverse biological functions of EGFR. Therefore, this study assumed that emodin in pancreatic cancer reverses EGFR inhibitor resistance by inhibiting Stat3 and that inhibition of EGFR and Stat3 synergistically exerted anticancer activity in pancreatic cancer. This hypothesis was further validated by various in vitro and in vivo experiments and its mechanism was elucidated. The implementation of this project will have a profound impact on overcoming the drug resistance of clinical drugs, and it is expected to discover new treatment strategies for pancreatic cancer.

\section{Materials and methods Cell culture}

Human pancreatic cancer cell lines PANC-1 and BxPC-3 were obtained from the Shanghai Institute of Cell Bank (Shanghai, China). The cells were maintained in DMEM medium (GibcoTM, 10564011) containing 10\% (v/v) FBS (GibcoTM, 10099141), $100 \mathrm{IU} / \mathrm{mL}$ penicillin and 100 $\mu \mathrm{g} / \mathrm{mL}$ streptomycin under $5 \% \mathrm{CO}_{2}$ water-saturated atmosphere at $37^{\circ} \mathrm{C}$. Cells were transfected with $1 \mu \mathrm{g}$ plasmid and $1.5 \mu \mathrm{l}$ Lipofectamine 2000 every well. The transfection efficiency can reach more than $70 \%$.

\section{Colony formation assay}

Colony formation assay was used to detect the capacity of cell proliferation. Briefly, 48 hrs after cell transfection, 100 $\mu \mathrm{L}$ PANC- 1 and BxPC- 3 cells $\left(5 \times 10^{4}\right.$ cells $\left./ \mathrm{mL}\right)$ were seeded into a 6 -well plate for 14 days. After being fixed with $10 \%$ formaldehyde for $20 \mathrm{mins}$, the forming colonies were then stained with $0.1 \%$ crystal violet (Sigma-Aldrich Co.).

\section{Cell transfection}

Cells were transfected with si-NC or si-Stat3. Twenty-four hours before transfection, the cells were inoculated into a 6 -well plate. When the confluence was reached to $50 \%$, 
Lipofectamine2000 (Invitrogen Inc., Carlsbad, CA, USA) was used to transfect the cells.

\section{Lentiviral vector packaging and stable cell line establishment}

Lv-NC (empty plasmid vector) and Lv-Stat3 (Stat3 over-expression lentiviral vector) used for cell transfection were purchased from Invitrogen Inc. (Carlsbad, CA, USA). Cells were collected in logarithmic growth phase. Then, the cell suspension was added into a 6-well plate $\left(2 \times 10^{6}\right.$ cells/well $)$ after being adjusted to appropriate density. About 3 days later after inoculation of cells, LV-transfected cells were screened using puromycin at less cell passage cultivation three times until plasmid is stably expressed. Cells were prepared for test in further studies after cell selection.

\section{The treatment of EFGR inhibitor in cell}

The EGFR inhibitor (erlotinib, gefitinib, afatinib and cetuximab) was used to treat the PANC-1 and BxPC-3 cells at the concentration of $20 \mathrm{nM}$. After $24 \mathrm{hrs}$ of incubation, cells were collected for further detection.

\section{Cell viability assay}

Cell proliferation assay was conducted using MTT Kit (11465007001, Sigma-Aldrich, St. Louis, USA). Briefly, cells $\left(1 \times 10^{4}\right.$ cells/well) were seeded into a 96 -well plate in triplicate. Following incubation at $37^{\circ} \mathrm{C}$ for different time $(12,24,48$ and $72 \mathrm{hrs})$ periods, the culture medium was removed and MTT reagent $(20 \mu \mathrm{L} ; 5 \mathrm{mg} / \mathrm{mL})$ was added and incubated at $37^{\circ} \mathrm{C}$ for another $4 \mathrm{hrs}$. Absorbance was measured at a wavelength of $490 \mathrm{~nm}$.

\section{Cell apoptosis analysis}

For apoptosis analysis, the cells were harvested following washing with ice-cold PBS. Then, the cells were stained with Annexin V-FITC apoptosis detection kit (556547, BD Biosciences, USA). Lastly, the apoptosis of stained cells was observed using a flow cytometer.

\section{qRT-PCR}

Total tissue and cell RNA were isolated using Trizol reagent (Invitrogen, Carlsbad, CA, USA) following the protocols. cDNA was then synthesized using the GoScript $^{\mathrm{TM}}$ Reverse Transcription kit (Madison, WI, USA). qRT-PCR was carried out according to the protocol of GoTaq ${ }^{\circledR}$ qRT-PCR Master mix kit (Madison, WI, USA).
GAPDH was included as an internal control. The sequences of primers were as follows: Stat3 forward primer: 5'- CAT CCT GAA GCT GAC CCAG G -3', reverse primer: 5'- TAT TGC TGC AGG TCG TTG GT-3'; EGFR forward primer: 5'- ACC AGC AAC AAT TCC ACC GT -3', reverse primer: 5'- CCA GAC AAG CCA CTC ACC AG-3', GAPDH forward primer: 5'- GAA AGC CTG CCG GTG ACT AA -3', reverse primer: 5'- GCA TCA CCC GGA GGA GAA AT - $3^{\prime}$.

\section{Western blot analysis}

After rinsed with PBS, the PC cells were lysed in extraction buffer for $15 \mathrm{mins}$, and then, $12,000 \mathrm{r} \mathrm{min}^{-1}$ is centrifuged at low temperature for 15 mins, and the supernatant is sucked and loaded into a new centrifuge tube. An Enhanced BCA Protein Concentration Assay Kit (P0009, Beyotime, Shanghai, China) was used to detect the protein concentration. Approximately $30 \mu \mathrm{g} /$ well protein was separated by $10 \%$ SDS-PAGE and then electro-transferred to PVDF membranes. Blots were blocked with 5\% BSA for $1.5 \mathrm{hrs}$ at $37^{\circ} \mathrm{C}$ followed by incubation overnight with the primary antibodies at $4^{\circ} \mathrm{C}$. After that, bands were incubated with peroxidase-conjugated secondary antibody for $1 \mathrm{hr}$ at room temperature and then visualized with Tanon 5200 Chemiluminescence imaging system. The amounts of the target proteins were analyzed using Image $\mathrm{J}$ analysis software and normalized to according control. Results were obtained from three independent experiments.

\section{In vivo tumor growth model}

All animal experiments were approved by the Animal Care and Ethics Committee of Wenzhou Medical University (WZU38869-582). The experimental procedures were complied with the Care and Use of Laboratory Animals from the National Institutes of Health. For tumor growth studies, BALB/c nude mice (4-6 weeks) (Vitalriver, Beijing, China) were subcutaneously injected with resuspended in Hank's buffer and mixed with an equal volume of matrigel (BD Biosciences) with $5 \times 10^{6}$ PANC-1 cells. When the tumors were approximately $30-50 \mathrm{~mm}^{3}$, the mice were randomly divided into 4 groups with 5 mice in each group: (1) saline (vehicle) orally daily for 4 weeks; (2) emodin with oral dose at $50 \mathrm{mg} / \mathrm{kg} /$ day for 4 weeks; (3) afatinib with oral dose at $50 \mathrm{mg} / \mathrm{kg} /$ day for 4 weeks; and (4) afatinib $(50 \mathrm{mg} / \mathrm{kg})$ and emodin $(50 \mathrm{mg} / \mathrm{kg})$ orally daily for 4 weeks. After 28 days, mice were sacrificed, and solid 
tumors were isolated for further analysis. And parts of tumors were evaluated by TUNEL staining.

\section{Statistical analysis}

All statistical results are displayed as mean \pm standard deviation and analyzed by ANOVA followed by Tukey's post hoc test among groups using SPSS Version 21.0 (Chicago, IL, USA). $P$-values less than 0.05 were considered statistically significant.

\section{Results}

\section{Emodin inhibits phosphorylation of Stat3} in pancreatic cancer cells

In order to confirm whether emodin can inhibit the phosphorylation of Stat3, emodin (final concentration 30-90 $\mu \mathrm{M})$ was used to treat pancreatic cancer cells. As shown in Figure 1, the date from Western blot showed that emodin effectively inhibited the phosphorylation of Stat3 in a dose-dependent manner.

\section{Emodin promotes apoptosis of pancreatic cancer cells}

As shown in Figure 2A, emodin significantly increased the apoptosis in PANC-1 and BxPC-3 cells. Further, the expressions of apoptosis-related proteins were detected. As shown in Figure 2B, the expressions of cleaved-caspase 3 and Bax-2 were significantly upregulated, while Bcl-2 expression was downregulated with the addition of emodin, especially in the high-dose group.

\section{Stat3 overexpression promotes proliferation of pancreatic cancer cells}

To evaluate the effect of Stat 3 on the proliferation of pancreatic cancer cells, the expression levels of Stat 3 in pancreatic cancer cells after Stat3 interfering RNA and Stat3 overexpression plasmid were determined. PCR and Western blot results showed that the expression of Stat 3 was significantly reduced by si-Stat3, while obviously increased by Stat3 overexpression plasmid (Figure $3 \mathrm{~A}$ and B). As shown in Figure 3C, high expression of Stat3 greatly increased the proliferation of PANC-1 and BxPC-3 cells.

\section{EGFR deletion promotes phosphorylation of Stat3 in pancreatic cancer cells}

Recent studies have shown that STAT3 and its mediated signaling pathway play an important role in EGFR-TKI resistance. ${ }^{24}$ Feedback activation of STAT3 is a novel mechanism for the development of EGFR inhibitor resistance. $^{25,26}$ To confirm the activation of EGFR inhibitors and Stat 3 in pancreatic cancer cells, p-Stat 3 expression in PANC-1 and BxPC-3 cells was detected after the addition of the selected four EGFR inhibitors. The results showed that EGFR inhibitors were effective in inhibiting EGFR expression (Figure 4A and B). Besides, the expression of p-stat 3 was significantly increased after EGFR inhibitors were added (Figure 4C).

\section{Emodin combined with EGFR inhibitor inhibits proliferation of pancreatic cancer cells in vitro}

To investigate whether emodin combined with EGFR inhibitors can synergistically exert antitumor effects, proliferation of pancreatic cancer cells in vitro was examined. The results showed that emodin combined with afatinib can greatly reduce the number of cell clones (Figure 5A). For further validation, MTT data also confirmed that emodin combined with afatinib significantly reduced the proliferation of pancreatic cancer cells (Figure 5B).
PANC-1

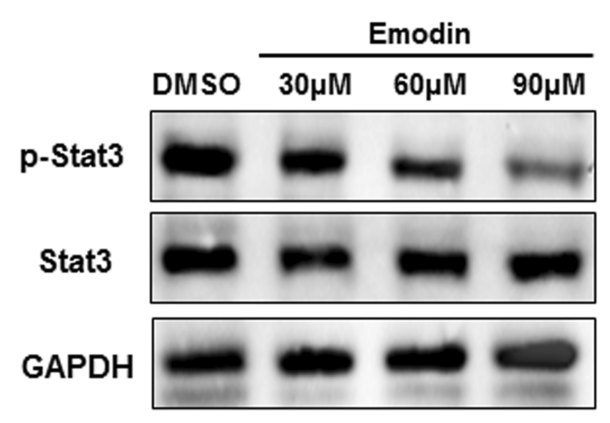

BxPC-3

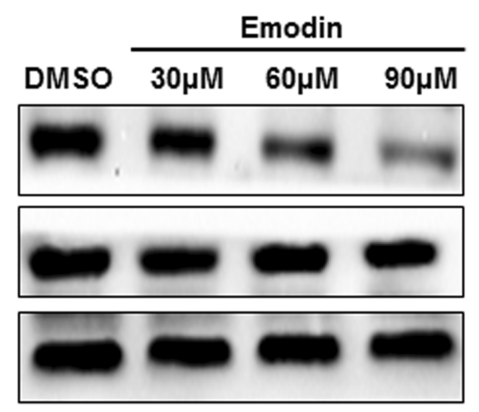

Figure I Emodin inhibits phosphorylation of Stat3 in pancreatic cancer cells. The PANC-I and BxPC-3 cells were incubated with emodin at the concentration of 30,60 or $90 \mu \mathrm{M}$. P-Stat3 expression in the cells was detected by Western blotting. 
A

PANC-1
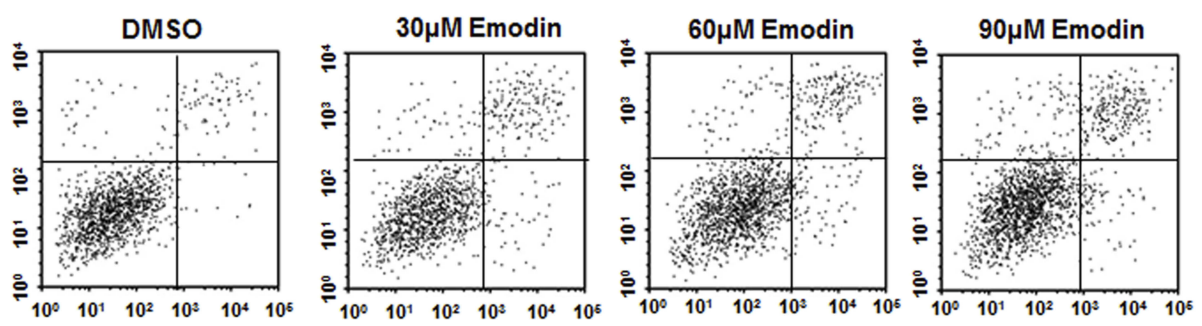

BxPC-3
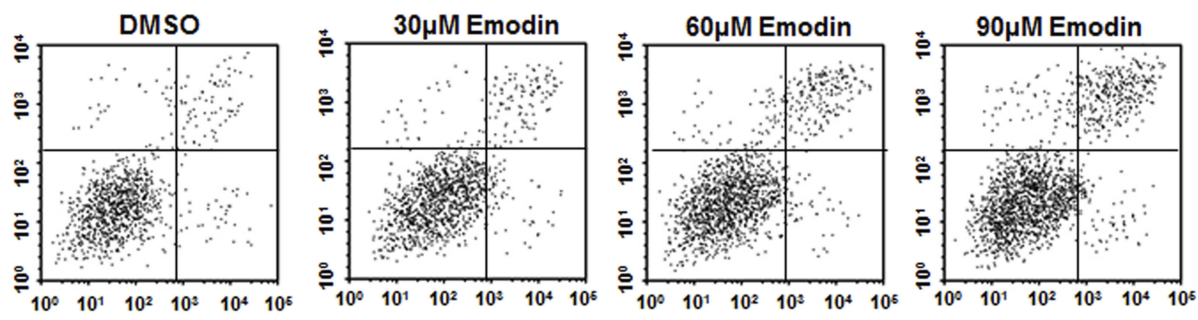

B
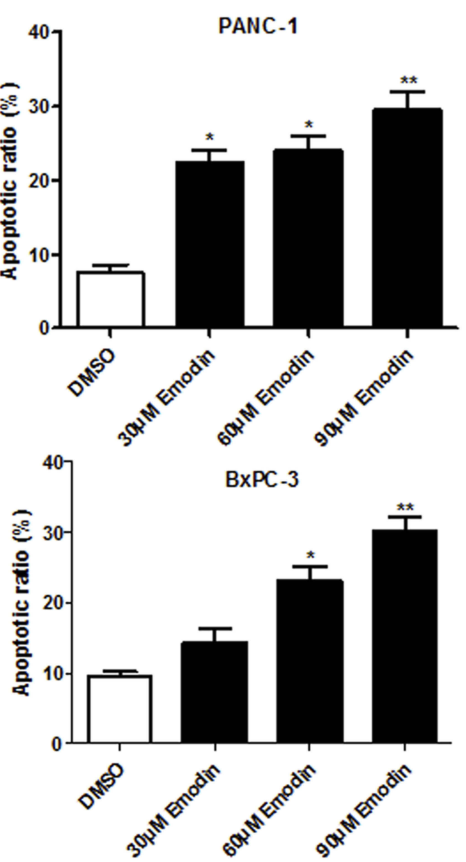

PANC-1

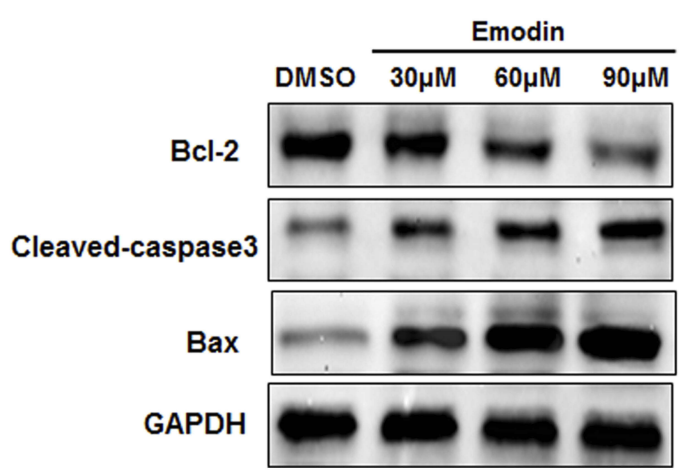

BxPC-3

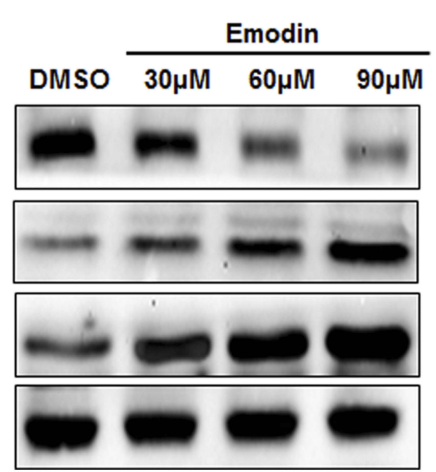

Figure 2 Emodin promotes apoptosis of pancreatic cancer cells. The PANC-I and BxPC-3 cells were incubated with emodin at the concentration of 30,60 or $90 \mu \mathrm{M}$. (A) Flow cytometry was used to detect the apoptosis of cells, and the right is the quantitative analysis of flow cytometry data. (B) Bcl-2, cleaved-caspase3, and Bax expressions in the cells were detected with Western blotting. $* P<0.05 ; * * P<0.01$.

\section{Emodin combined with EGFR inhibitor inhibits proliferation of pancreatic cancer cells in vivo}

In order to better evaluate the antitumor effect of emodin combined with EGFR inhibitors in vivo, tumor-forming experiments in nude mice were performed and the results revealed that emodin combined with EGFR inhibitors effectively inhibited tumor growth (Figure 6A-C). Western blot analysis showed that emodin combined with afatinib exerted a stronger EGFR inhibitory effect (Figure 6D). Meanwhile, we also detected the protein expression of $\mathrm{p}$-Stat3, and the results demonstrated that emodin combined with EGFR inhibitor effectively increased the expression of p-Stat3 (Figure 6E). And the TUNEL assay showed that emodin combined with EGFR inhibitors promotes cell apoptosis in the tumor tissue more effectively (Figure 6F).

\section{Discussion}

Although most antitumor drugs have certain curative effects, they still have many disadvantages such as side effects and high prices. ${ }^{27}$ Traditional Chinese medicines and natural medicines have small toxic and side effects and diverse structure, making it an important 
B

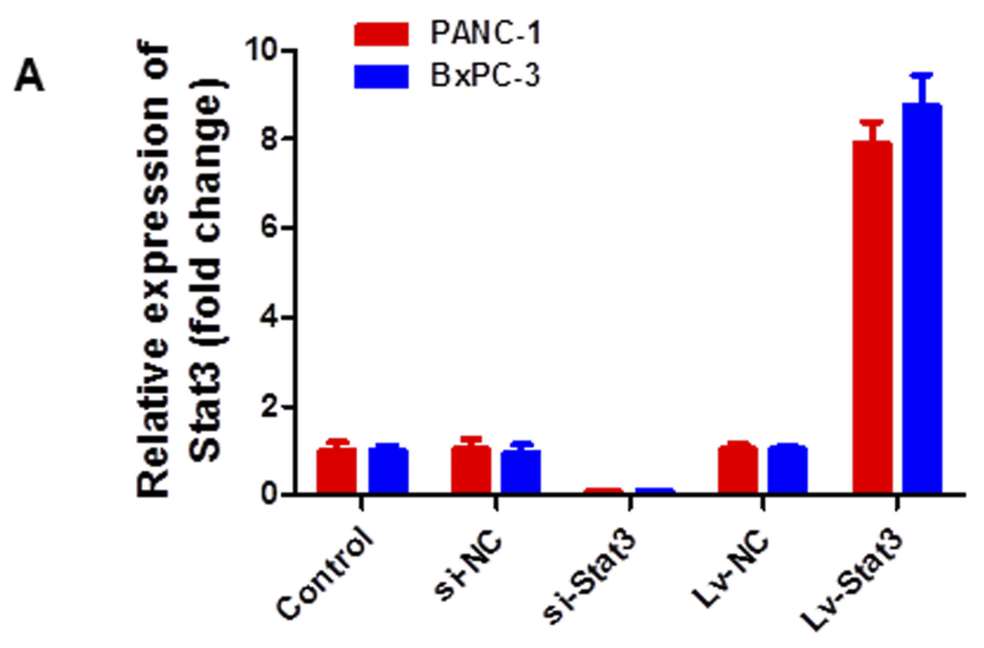

PANC-1

\section{BxPC-3}

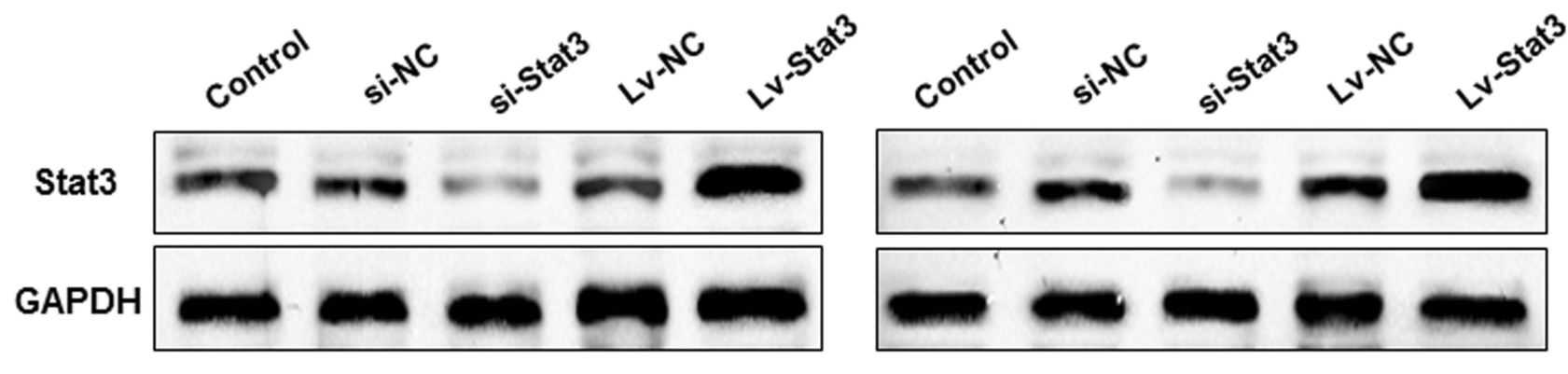

C

PANC-1

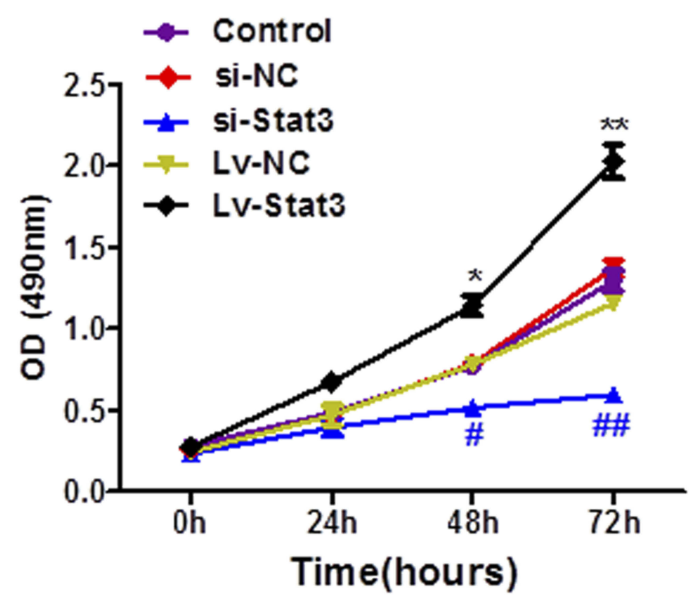

BXPC-3

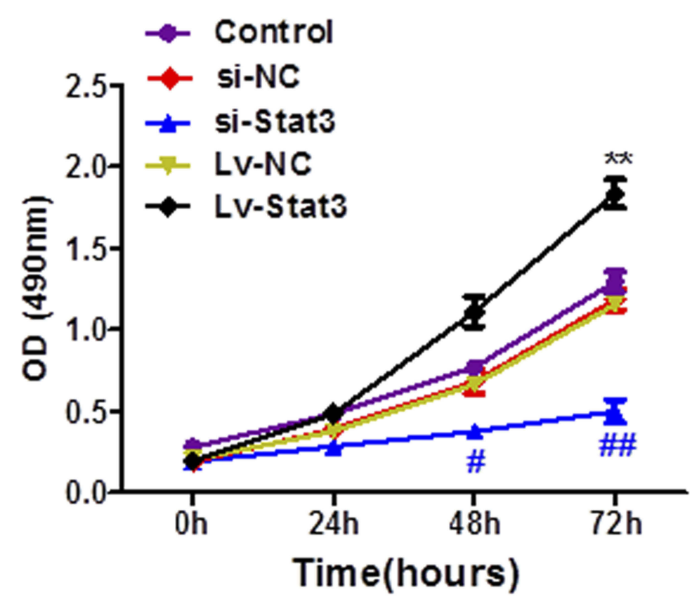

Figure 3 The overexpression of Stat3 promotes proliferation of pancreatic cancer cells. (A) The mRNA and (B) protein expression of Stat3 were detected with qRT-PCR and Western blotting, respectively. (C) The proliferation of cells was detected with MTT assay.

way to find antitumor active ingredients from natural products in recent years. ${ }^{28}$ Currently, $60 \%$ of the antitumor drugs used clinically are derived from extremely abundant natural products. ${ }^{29}$ Emodin is a terpenoid monomer extracted from rhubarb, which obviously induced the apoptosis of pancreatic cancer cells but has little effect on normal cell proliferation. ${ }^{18}$ Emodin is a new class of antitumor drugs whose molecular mechanism in the Stat3 signaling pathway remains unclear. One of the innovations of this project is to confirm that the direct target of emodin is Stat3, which exerts antitumor effect by inhibiting Stat3. 
B

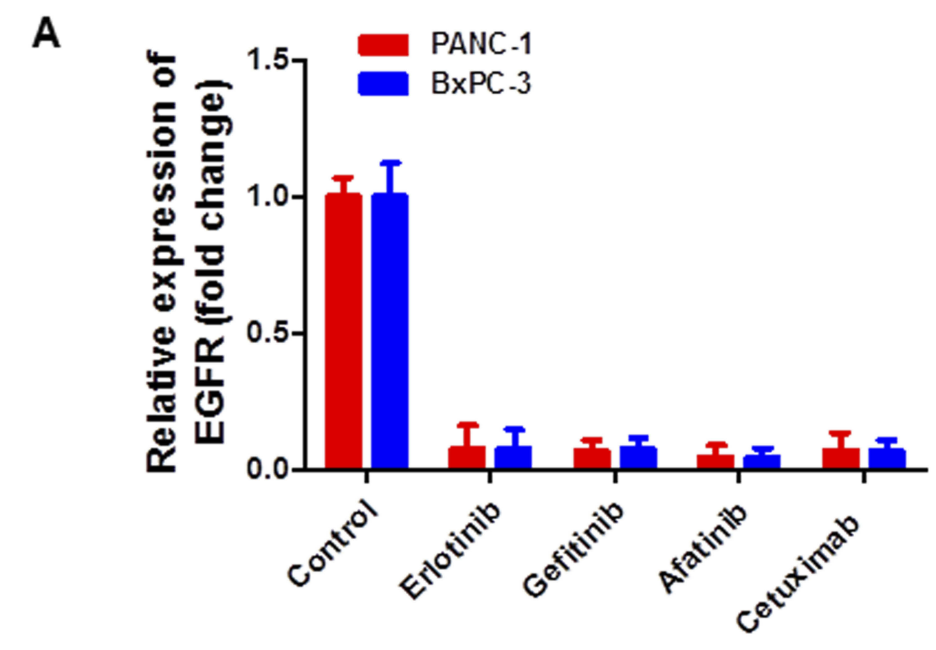

PANC-1

BxPC-3

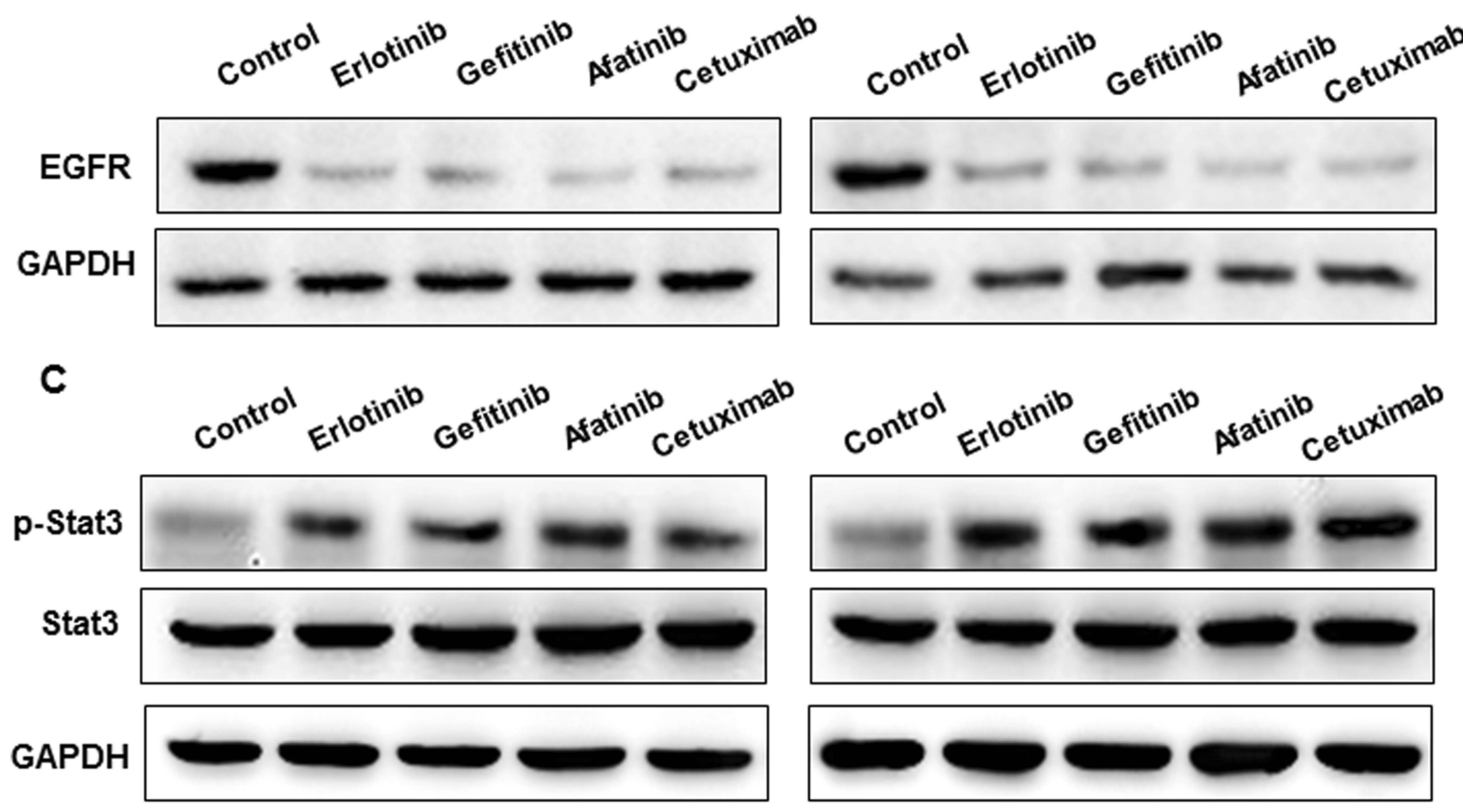

Figure 4 EGFR inhibitor promotes phosphorylation of Stat3 in pancreatic cancer cells. The EGFR inhibitor (erlotinib, gefitinib, afatinib and cetuximab) was used to treat the PANC-I and BxPC-3 cells at the concentration of $20 \mathrm{nM}$. (A) The mRNA and (B) protein expressions of EGFR were detected with qRT-PCR and Western blotting, respectively. (C) And the protein expression of $\mathrm{p}$-Stat3 was detected with Western blotting.

EGFR belongs to the tyrosine kinase receptor (RTK) family, and over-activation of EGFR promotes the cascade response of downstream signaling pathways, including Ras/Raf/MEK/ERK, PI3K-AKT and JAK/STAT. ${ }^{30,31}$ This leads to the occurrence and metastasis of the tumor. EGFR inhibitors have been widely used in clinical oncology treatment, but their toxic side effects and drug resistance have greatly limited their application.

Many studies have found that natural products combined with chemical drugs have special effects. For example, methyl- $\beta$-cyclodextrin sensitizes $\mathrm{MCF}-7$ and Hepa1-6 cells to doxorubicin. Tumor growth was retarded and survival increased in mice that were administered methyl- $\beta$-cyclodextrin together with doxorubicin to as compared to either agent alone. ${ }^{32}$ Combined with modern experimental research, emodin has antitumor effect with low toxicity and has synergistic effect with various antitumor drugs, which is a potential effective drug for clinical tumor treatment. ${ }^{33}$ Therefore, we aimed at emodin which is a natural product. Our 

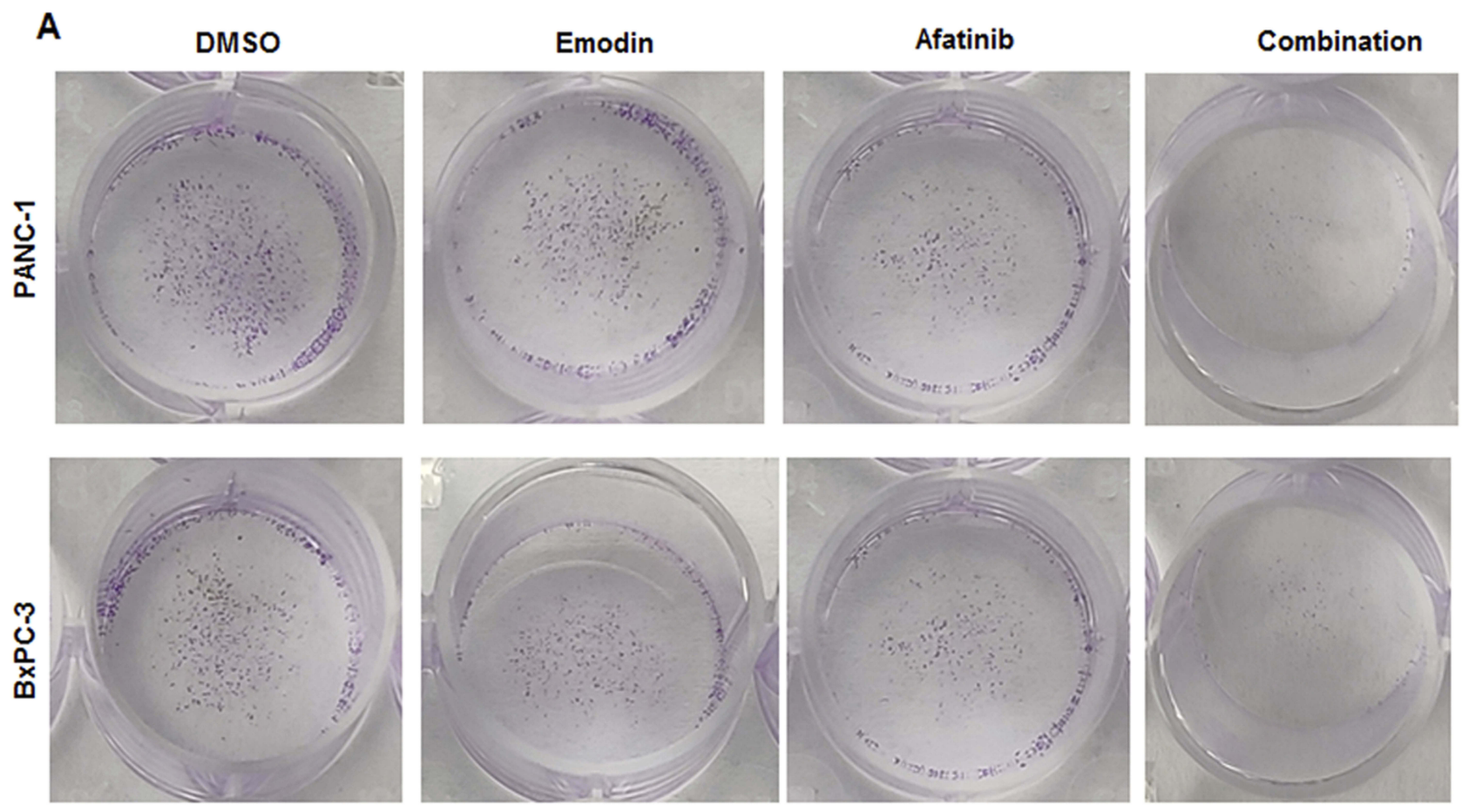

B

\section{PANC-1}

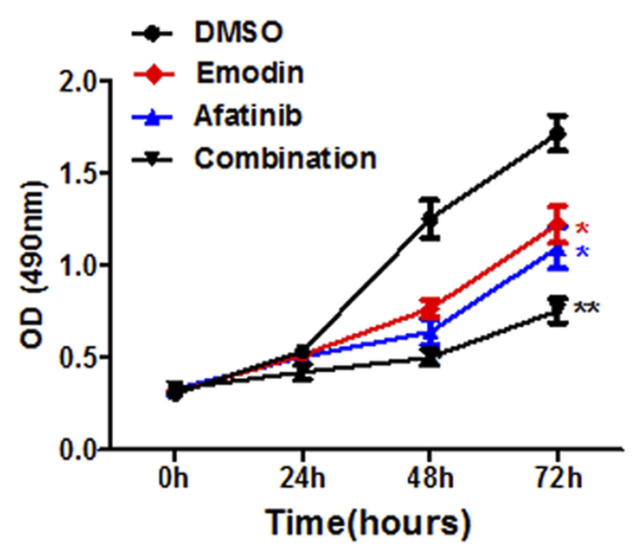

BXPC-3

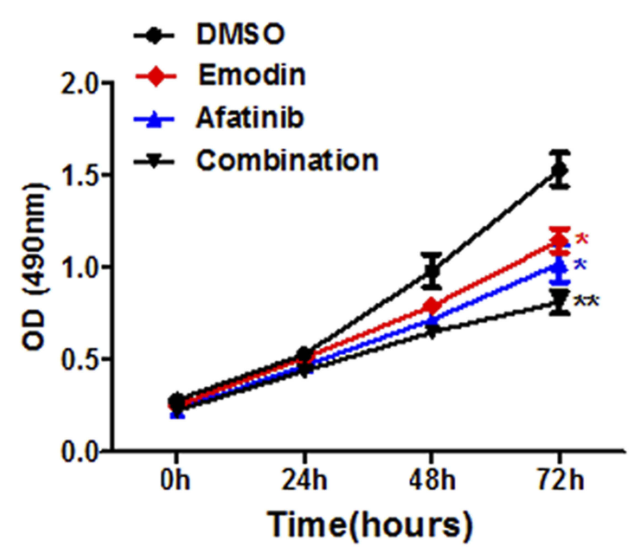

Figure 5 Emodin combined with EGFR inhibitor inhibits proliferation of pancreatic cancer cells in vitro. (A) The colon and (B) MTT assays were used to detect the proliferation in PANC-I and BxPC-3 cells.

research found that emodin combined with the EGFR inhibitor afatinib has a stronger inhibitory activity against pancreatic cancer cells compared with emodin or EGFR inhibitor alone. These results indicated that the combination of emodin and EGFR inhibitors for antitumor had the potential to reverse the resistance of EGFR inhibitors, possibly with regulatory, synergistic or additive effects.

The resistance mechanism of EGFR inhibitors is related to EGFR secondary mutation, abnormal activation of other RTKs, changes in signal pathway components or regulatory factor gene expression, internalization of EGFR and changes in tumor microenvironment. ${ }^{34}$ As an important downstream regulator of EGFR signaling pathway, Stat3 was involved in EGFR-mediated migration and invasion of cancer cells. By studying the interaction between Stat3 and EGFR signaling pathway, the mechanism of drug resistance in tumor therapy can be further revealed, and more effective antitumor drugs can be developed. It has been found that the EGFR inhibitors erlotinib, afatinib, and dacomitinib acted on lung cancer cells to activate the IL-6/JAK/Stat3 signaling pathway, which in turn induced 
A

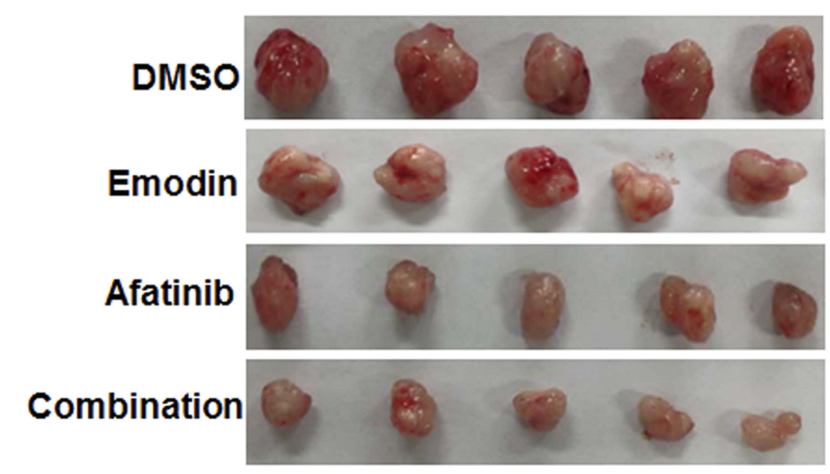

B

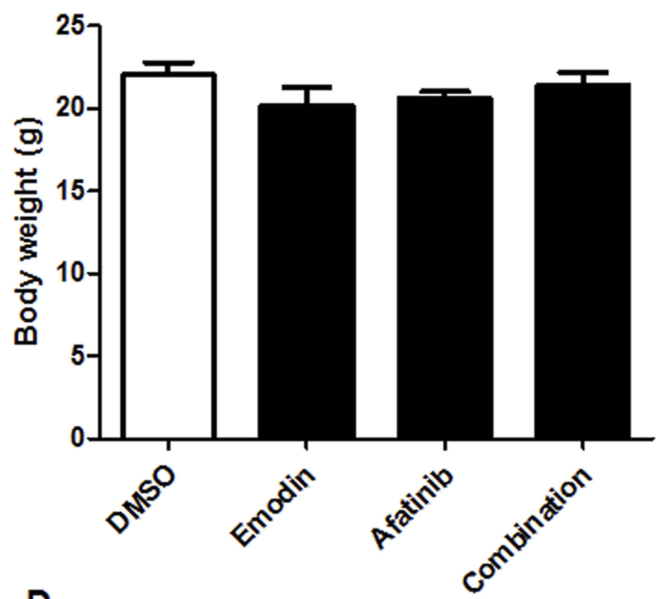

D

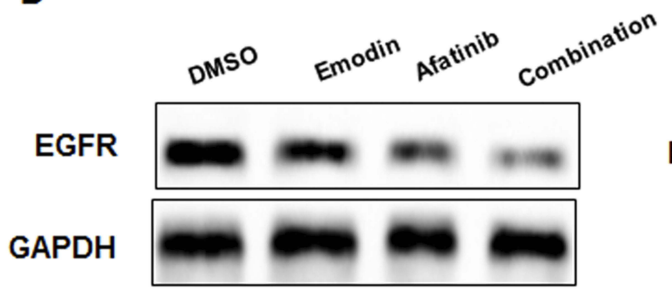

C

E
B
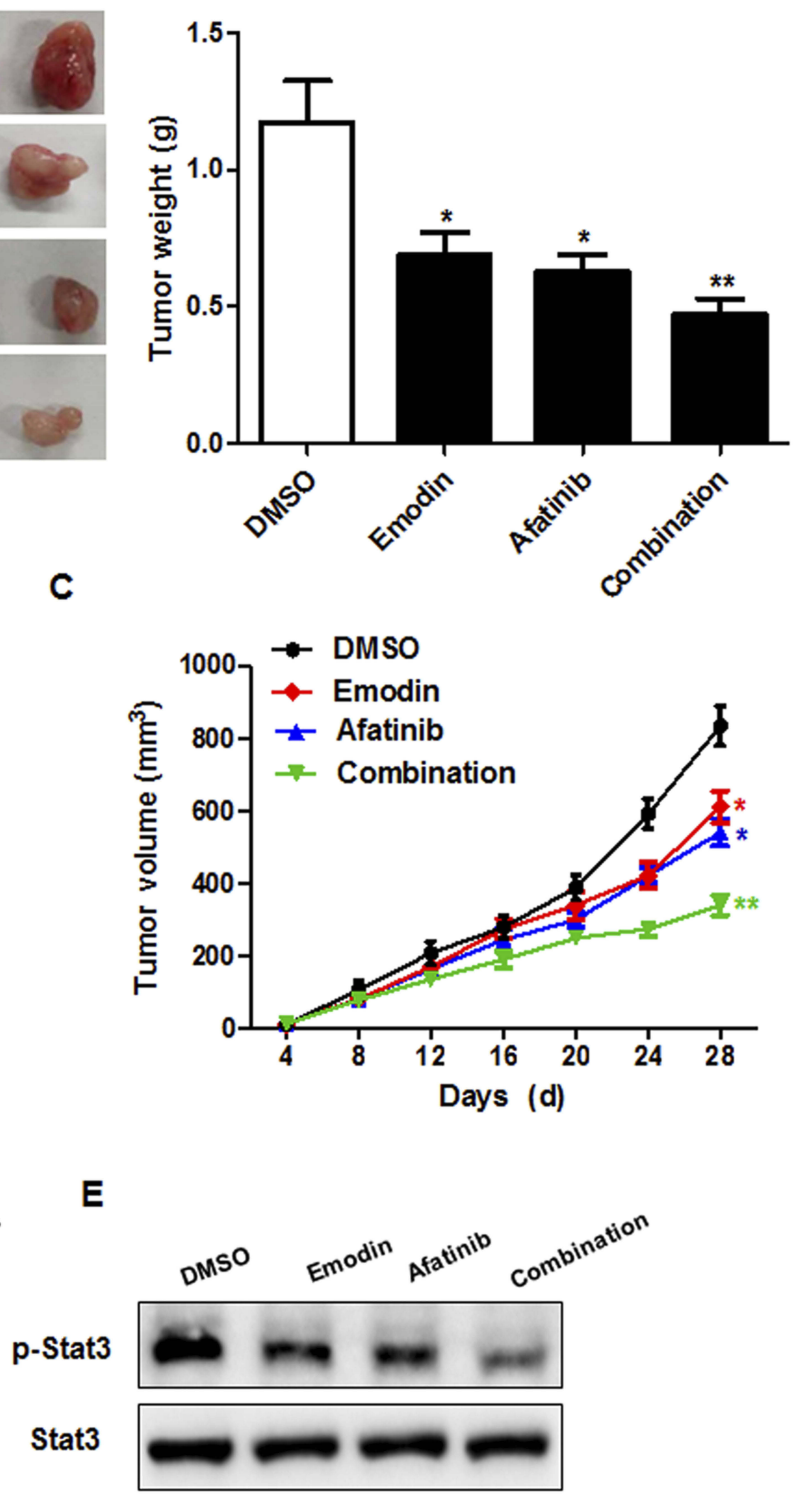

GAPDH

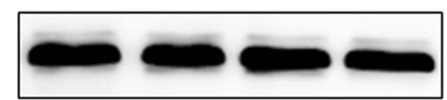

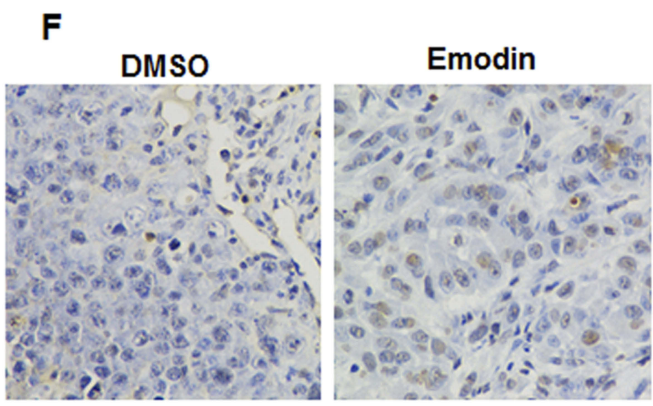

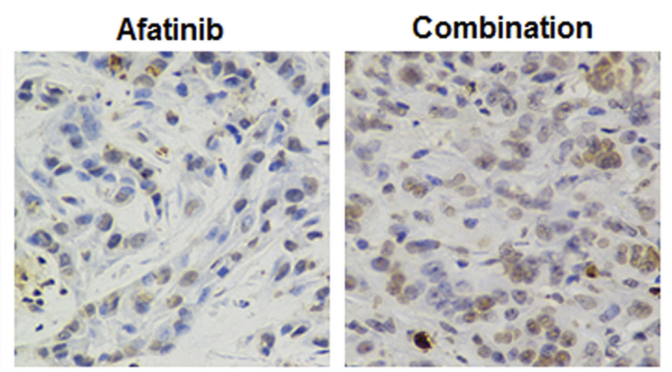

Figure 6 Emodin combined with EGFR inhibitor inhibits proliferation of pancreatic cancer cells in vivo. Mice were administrated with vehicle (saline), emodin at 50 mg/kg/ day, afatinib at $50 \mathrm{mg} / \mathrm{kg} / \mathrm{day}$, or the combination of the 2 drugs for 4 weeks. Tumor size and tumor weight (A), body weight (B) and tumor volume (C) of mice were measured. The protein of EGFR (D) and $\mathrm{p}-\mathrm{Stat} 3(\mathbf{E})$ were detected with Western blotting. TUNEL assay detected the apoptosis in the tumor tissues $(\mathbf{F})$. $* P<0.05$; $* * P<0.0$ I. 
drug resistance. ${ }^{35}$ When EGFR inhibitor cetuximab is administered to metastatic colorectal cancer, its resistance is also associated with Stat 3 activation. ${ }^{36}$ Therefore, feedback activation of the Stat 3 signaling pathway is a novel mechanism of EGFR inhibitor resistance, which can be reversed by the inhibition of Stat3.

Although modern research has a certain understanding of the mechanism of the antitumor effect of emodin, it still needs to be further studied. We found that emodin inhibited the level of p-Stat 3 in pancreatic cancer cells and inhibited its proliferation and promoted apoptosis. Through MTT experiments, when emodin was applied to human pancreatic cancer cells, the inhibition rate of these three cells was elevated with the increase of emodin concentration. The $\mathrm{p}$-Stat 3 protein expression was detected at different concentrations of emodin after interfering with PANC-1 and BxPC-3 cells for 24 hrs. And emodin treatment decreased the $\mathrm{p}-\mathrm{Stat} 3$ protein expression in a concentration-dependent manner. Additionally, the expression of apoptosis-inhibiting gene Bcl-2 was decreased, but cleaved-caspase3 expression was increased, indicating that emodin inhibited the cell growth and promoted the apoptosis by inhibiting phosphorylation of Stat3 and affecting apoptosis-related proteins. Meanwhile, in vivo and in vitro experiments showed that emodin reversed the resistance of EGFR inhibitors in pancreatic cancer. When emodin was combined with EGFR inhibitor afatinib, the inhibitory activity against pancreatic cancer cells was better than that of emodin or EGFR inhibitor alone.

Based on these findings, we hypothesized that in pancreatic cancer, emodin reverses EGFR inhibitor resistance by inhibiting Stat3 and that emodin and EGFR inhibitors synergistically exert antitumor effects. Our research elucidated the antitumor effect of emodin by inhibiting Stat 3 and verified that EGFR inhibitor resistance in pancreatic cancer was associated with Stat3 activation. It provided a new theoretical basis and idea for solving EGFR inhibitor resistance.

\section{Acknowledgment}

The project was supported by Natural Science Foundation of Zhejiang project (LQ18H290003 and Y19H290009) and Wenzhou Science and Technology Bureau planning project (Y20190199, Y20160143).

\section{Disclosure}

The authors report no conflicts of interest in this work.

\section{References}

1. Xia L, Song M, Sun M, Chen W, Yang C. miR-486 promotes Capan2 pancreatic cancer cell proliferation by targeting phosphatase and tensin homolog deleted on chromosome 10 (PTEN). Front Genet. 2019;10:541. doi:10.3389/fgene.2019.00541

2. Li H, Zhang SR, Xu HX, et al. SRPX2 and RAB31 are effective prognostic biomarkers in pancreatic cancer. J Cancer. 2019;10 (12):2670-2678. doi:10.7150/jca.32072

3. Zhou Y, Cui J, Du H. Autoantibody-targeted TAAs in pancreatic cancer: a comprehensive analysis. Pancreatol. 2019;19(5):760-768.

4. Lee B, Lipton L, Cohen J, et al. Circulating tumor DNA as a potential marker of adjuvant chemotherapy benefit following surgery for localised pancreatic cancer. Ann Oncol. 2019;28.doi:10.1093/annonc/ mdz200

5. Thomas NA, Abraham RG, Dedi B, Krucher NA. Targeting retinoblastoma protein phosphorylation in combination with EGFR inhibition in pancreatic cancer cells. Int J Oncol. 2019;54(2):527-536.

6. Ishimoto U, Kinoshita A. The efficacy and safety of nab paclitaxel plus gemcitabine in elderly patients over 75 years with unresectable pancreatic cancer compared with younger patients. Cancer Chemother Pharmacol. 2019; 84(3):647-654. doi:10.10 07/s00280-019-03895-2

7. Li Z, Wang M, Yao X, et al. Development of a novel EGFR-targeting antibody-drug conjugate for pancreatic cancer therapy. Target Oncol. 2019;14(1):93-105. doi:10.1007/s11523-018-0616-8

8. Wang Q, Yang S, Wang K, Sun SY. MET inhibitors for targeted therapy of EGFR TKI-resistant lung cancer. J Hematol Oncol. 2019;12(1):63. doi:10.1186/s13045-019-0759-9

9. Lee K, Kim Y, Jung HA, et al. Repeat biopsy procedures and T790M rates after afatinib, gefitinib, or erlotinib therapy in patients with lung cancer. Lung Cancer. 2019;130:87-92. doi:10.1016/j.lungcan.2019. 01.012

10. Qiu W, Zhang C, Wang S, et al. A novel anti-EGFR mAb Ame55 with lower toxicity and better efficacy than cetuximab when combined with irinotecan. J Immunol Res. 2019;2019:3017360.

11. Muhammad N, Steele R, Isbell TS, Philips N, Ray RB. Bitter melon extract inhibits breast cancer growth in preclinical model by inducing autophagic cell death. Oncotarget. 2017;8(39):66226-66236. doi:10.18632/oncotarget.19887

12. Bhattacharya S, Muhammad N, Steele R, Peng G, Ray RB. Immunomodulatory role of bitter melon extract in inhibition of head and neck squamous cell carcinoma growth. Oncotarget. 2016;7 (22):33202-33209. doi:10.18632/oncotarget.v7i22

13. Evans L, Ferguson B. Emodin attenuates pathological cardiac hypertrophy by regulating gene expression through acetyl-histonemediated actions (P06-036-19). Current Dev Nutr. 2019;3(Suppl 1). doi:10.1093/cdn/nzz031.P06-036-19

14. Li Y, Xiong W, Yang J, et al. Attenuation of inflammation by emodin in lipopolysaccharide-induced acute kidney injury via inhibition of tolllike receptor 2 signal pathway. Iran J Kidney Dis. 2015;9(3):202-208.

15. Xie R, Liu M, Li S. Emodin weakens liver inflammatory injury triggered by lipopolysaccharide through elevating microRNA-145 in vitro and in vivo. Artif Cells Nanomed Biotechnol. 2019;47(1):18771887. doi:10.1080/21691401.2019.1614015

16. Braumann C, Koplin G. Dose-dependent role of novel agents emodin and BTB14431 in colonic cancer treatment in rats. Acta Chir Belg. 2017;117 (6):376-384.

17. Gao R, Wu X, Huang Z, et al. Anti-tumor effect of aloe-emodin on cervical cancer cells was associated with human papillomavirus E6/ E7 and glucose metabolism. Onco Targets Ther. 2019;12:3713-3721. doi:10.2147/OTT.S182405

18. Chen H, Wei W, Guo Y, et al. Enhanced effect of gemcitabine by emodin against pancreatic cancer in vivo via cytochrome $\mathrm{C}$-regulated apoptosis. Oncol Rep. 2011;25(5):1253-1261. 
19. Ma JW, Hung CM, Lin YC, Ho CT, Kao JY, Way TD. Aloe-emodin inhibits HER-2 expression through the downregulation of Y-box binding protein-1 in HER-2-overexpressing human breast cancer cells. Oncotarget. 2016;7(37):58915-58930.

20. Chen KC, Juang SH, Lien JC. Identification of antiproliferative emodin analogues as inhibitors of epidermal growth factor receptor in cancer. Int J Mol Med. 2019;43(3):1281-1288.

21. Saunders IT, Mir H, Kapur N, Singh S. Emodin inhibits colon cancer by altering BCL-2 family proteins and cell survival pathways. Cancer Cell Int. 2019;19:98. doi:10.1186/s12935-019-0820-3

22. Lin SZ, Xu JB, Ji X, et al. Emodin inhibits angiogenesis in pancreatic cancer by regulating the transforming growth factorbeta/drosophila mothers against decapentaplegic pathway and angiogenesis-associated microRNAs. Mol Med Rep. 2015;12 (4):5865-5871. doi:10.3892/mmr.2015.4158

23. Juan L, Peishu L, Hongluan M, Ancong W, Xiaolei Z. Emodin sensitizes paclitaxel-resistant human ovarian cancer cells to paclitaxel-induced apoptosis in vitro. Oncol Rep. 2009;21(6):1605-1610.

24. Yang L, Lin S, Kang Y, et al. Rhein sensitizes human pancreatic cancer cells to EGFR inhibitors by inhibiting STAT3 pathway. $J$ Exp Clin Cancer Res. 2019;38(1):31. doi:10.1186/s13046-018-1015-9

25. Zhao C, Li H, Lin HJ, Yang S, Lin J, Liang G. Feedback activation of STAT3 as a cancer drug-resistance mechanism. Trends Pharmacol Sci. 2016;37(1):47-61. doi:10.1016/j.tips.2015.10.001

26. Shi L, Zheng $\mathrm{H}, \mathrm{Hu} \mathrm{W}$, et al. Niclosamide inhibition of STAT3 synergizes with erlotinib in human colon cancer. Onco Targets Ther. 2017;10:1767-1776. doi:10.2147/OTT.S129449

27. Norum OJ, Asv F, Weyergang A, Golab J, Berg K. Photochemical delivery of bleomycin induces T-cell activation of importance for curative effect and systemic anti-tumor immunity. $J$ Controlled Release Off J Controlled Release Soc. 2017;268:120-127. doi:10.1016/j.jconrel.2017.10.015
28. Man S, Gao W, Wei C, Liu C. Anticancer drugs from traditional toxic Chinese medicines. Phytother Res. 2012;26(10):1449-1465.

29. Ii F. Anti-tumor natural product research focused on plants found in the Southern Appalachian region. [Theses]. Western Carolina University. 2010.

30. Coley HM, Shotton CF, Ajose-Adeogun A, Modjtahedi H, Thomas H. Receptor tyrosine kinase (RTK) inhibition is effective in chemosensitising EGFR-expressing drug resistant human ovarian cancer cell lines when used in combination with cytotoxic agents. Biochem Pharmacol. 2006;72(8):941-948. doi:10.1016/j.bcp.2006.07.022

31. Torres AF, Nogueira C, Magalhaes J, et al. Expression of EGFR and molecules downstream to PI3K/Akt, Raf-1-MEK-1-MAP (Erk1/2), and JAK (STAT3) pathways in invasive lung adenocarcinomas resected at a single institution. Anal Cell Pathol Cell Oncol. 2014;2014:352925.

32. Mohammad N, Singh SV, Malvi P, et al. Strategy to enhance efficacy of doxorubicin in solid tumor cells by methyl-beta-cyclodextrin: involvement of p53 and Fas receptor ligand complex. Sci Rep. 2015;5:11853. doi:10.1038/srep11853

33. Guo J, Li W, Shi H, et al. Synergistic effects of curcumin with emodin against the proliferation and invasion of breast cancer cells through upregulation of miR-34a. Mol Cell Biochem. 2013;382(1-2):103-111. doi:10.1007/s11010-013-1723-6

34. Lihua H, Liwu F. Mechanisms of resistance to EGFR tyrosine kinase inhibitors. Acta Pharm Sinica B. 2015;5(5):390-401. doi:10.1016/j. apsb.2015.07.001

35. Zhang N, Zeng Y, Du W, et al. The EGFR pathway is involved in the regulation of PD-L1 expression via the IL-6/JAK/STAT3 signaling pathway in EGFR-mutated non-small cell lung cancer. Int J Oncol. 2016;49(4):1360. doi:10.3892/ijo.2016.3632

36. Ung N, Putoczki TL, Stylli SS, et al. Anti-EGFR therapeutic efficacy correlates directly with inhibition of STAT3 activity. Cancer Biol Ther. 2014;15(5):623-632. doi:10.4161/cbt.28179

\section{Publish your work in this journal}

Cancer Management and Research is an international, peer-reviewed open access journal focusing on cancer research and the optimal use of preventative and integrated treatment interventions to achieve improved outcomes, enhanced survival and quality of life for the cancer patient.
The manuscript management system is completely online and includes a very quick and fair peer-review system, which is all easy to use. Visit http://www.dovepress.com/testimonials.php to read real quotes from published authors. 